\title{
Value of diffusion-weighted imaging for evaluating chemotherapy response in osteosarcoma: A meta-analysis
}

\author{
TADAHIKO KUBO, TAISUKE FURUTA, MUHAMMAD P. JOHAN, MITSUO OCHI and NOBUO ADACHI \\ Department of Orthopaedic Surgery, Graduate School of Biomedical Sciences, \\ Hiroshima University, Hiroshima 734-8551, Japan
}

Received November 3, 2016; Accepted March 29, 2017

DOI: $10.3892 /$ mco.2017.1273

\begin{abstract}
The histological examination of the tumor necrosis upon surgery remains the most reliable prognostic factor for osteosarcoma. However, the detection of more early prognostic factors is desirable in order to increase the survival rates and decrease the risk rates for iatrogenic toxicity. The purpose of the current systematic review and meta-analysis was to provide an up-to-date summary of the role of diffusion-weighted imaging (DWI) for the preoperative assessment of the chemotherapy response in osteosarcoma. Articles evaluating DWI for the preoperative assessment of the chemotherapy response of osteosarcoma were systematically searched for in four electronic literature databases. The mean difference in apparent diffusion coefficient (ADC) following neoadjuvant chemotherapy between good and poor histological responders was assessed in 5 studies. The mean difference in the ADC ratio (the percentage change in ADC between post-neoadjuvant and pre-neoadjuvant chemotherapy) reported in 3 studies was also assessed. Five articles with 106 patients fulfilled all of the inclusion criteria for the meta-analysis. Significant mean differences were found between good and poor responders in the ADC in the 5 studies $(\mathrm{P}=0.03)$ and the ADC ratio in the 3 studies $(\mathrm{P}<0.00001)$. The good responders demonstrated a higher ADC and a higher ADC ratio than the poor responders. DWI performed with ADC values was useful for predicting the chemotherapeutic response of osteosarcoma. This method may have promising potential as a preoperative non-invasive modality.
\end{abstract}

\section{Introduction}

Pre and postoperative chemotherapy in addition to surgery have significantly increased the survival rate for patients with osteosarcoma $(1,2)$. However, over the last 20 years, attempts at more intense chemotherapeutic therapy using conventional

Correspondence to: Dr Tadahiko Kubo, Department of Orthopaedic Surgery, Graduate School of Biomedical Sciences, Hiroshima University, 1-2-3 Kasumi, Minami-ku, Hiroshima 734-8551, Japan E-mail: kubot@hiroshima-u.ac.jp

Key words: meta-analysis, diffusion-weighted imaging, apparent diffusion coefficient, osteosarcoma, chemotherapy response cancer agents have not improved the survival rate significantly. Furthermore, in spite of an aggressive surgical and chemotheraputic treatment strategy, patients with unresectable primary osteosarcoma and those with distant metastases still have a poor prognosis (3-5). The prognosis strongly correlates with the tumor histological response to preoperative chemotherapy in osteosarcoma $(6,7)$. However, this valuable standard criterion is available only following surgery, which means that histological evaluation of tumor necrosis during the course of chemotherapy requires repeated invasive biopsies. The quantitative evaluation of preoperative radiological changes using diffusion-weighted imaging (DWI), dynamic magnetic resonance imaging, thallium-201 scintigraphy and positron emission tomography with computed tomography (PET/CT) has been challenged (8-12). The operative treatment and neoadjuvant chemotherapy of suspected poor responders may then be intensified earlier, potentially increasing their survival rates and decreasing the risk rates of iatrogenic toxicity.

DWI is currently the only imaging method to non-invasively measure the local diffusion characteristics of water molecules in vivo. It is able to reflect the spatial composition and the functional status of water exchange among various tissues in pathophysiological states from the molecular level. The apparent diffusion coefficient (ADC) is used to measure water diffusion and has a decreasing tendency in highly cellular tissue. DWI has been used to classify the subtype of musculoskeletal tumors (13-16). As the signal of water diffusion is directly associated with the tumor cellularity, necrotic areas in the tumor increase a local diffusion signal. This phenomenon has been demonstrated in clinical and experimental practice (17-20). Although the ADC value on DWI may be a promising tool, due to the scant data currently available, there is no routine practice for DWI to predict the chemotherapeutic response of osteosarcoma.

The objective of the present study was to provide an up-to-date summary of the role of DWI for the preoperative assessment of the chemotherapy response of osteosarcoma. The mean difference of post-neoadjuvant chemotherapy ADC between good and poor histological responders of osteosarcoma was assessed using a systematic literature search and meta-analysis.

\section{Materials and methods}

Literature search. A systematic literature search was performed following the Preferred Reporting Items for 
Systematic Reviews and Meta-Analyses (PRISMA) statement (21). The main research question, consisting of the Target Population (including previous tests), Index Test, Comparator Test, Outcome and Study design (PICOS) strategy, was formulated into a search query. A combination of the terms 'diffusion-weighted imaging' and 'osteosarcoma' was searched without a time limitation, on four electric literature databases: MEDLINE, EMBASE, Web of Science and Cochrane Library.

Study selection. Two reviewers (TF and MJP) evaluated potentially relevant articles for eligibility. The decision of article inclusion or exclusion was hierarchical and firstly made on the basis of the article title, then of the article abstract and finally of the whole article. If either reviewer judged the article title and subsequently the article abstract to be potentially eligible, the two reviewers independently evaluated the whole article for eligibility using predetermined inclusion or exclusion criteria.

The inclusion criteria were i) articles published in English; ii) diffusion-weighted imaging was used to predict histological response following preoperative chemotherapy in osteosarcoma; iii) All ADC values or the mean ADC values were described; and iv) when parts of data were presented in more than one article, the most recent article was used.

Data extraction. The same investigators independently reviewed the included articles in consensus to extract study information for the meta-analysis.

Quality evaluation. The quality of study designs was assessed using the Quality Assessment of Diagnostic Accuracy Studies-2 (QUADAS-2) tool (22).

Meta-analysis. The mean difference in ADC value following neoadjuvant chemotherapy between good and poor histological responders was assessed in the 5 studies. Additionally, the ADC ratio was calculated by using the following formula to assess the relative change in the pre- and post-neoadjuvant chemotherapy ADC values of osteosarcomas: $\mathrm{ADC}$ ratio=(postchemotherapy ADC-prechemotherapy ADC)/prechemotherapy ADC x100. The mean difference in ADC ratio was correlated between good and poor histological responders in 3 of the 5 studies. Heterogeneity of the mean difference of each study was evaluated using the inconsistency index I-square $\left(I^{2}\right)$ test as well as the $\chi^{2}$ test. An $I^{2}>50 \%$ and/or $\mathrm{P}<0.10$ was considered to be statistically significant. The Der Simonian and Laird random effect model was applied if significant heterogeneity between studies was observed, while a fixed effect model was used in the absence of significant between-study heterogeneity. Publication bias was estimated using funnel plot asymmetry tests. All meta-analysis was performed using Review Manager software, version 5 (Cochrane Collaboration, Oxford, UK). P<0.05 was considered to indicate a statistically significant difference.

\section{Results}

Literature search and study selection. The PICOS main research question was $\mathrm{P}$, patients with osteosarcoma treated by

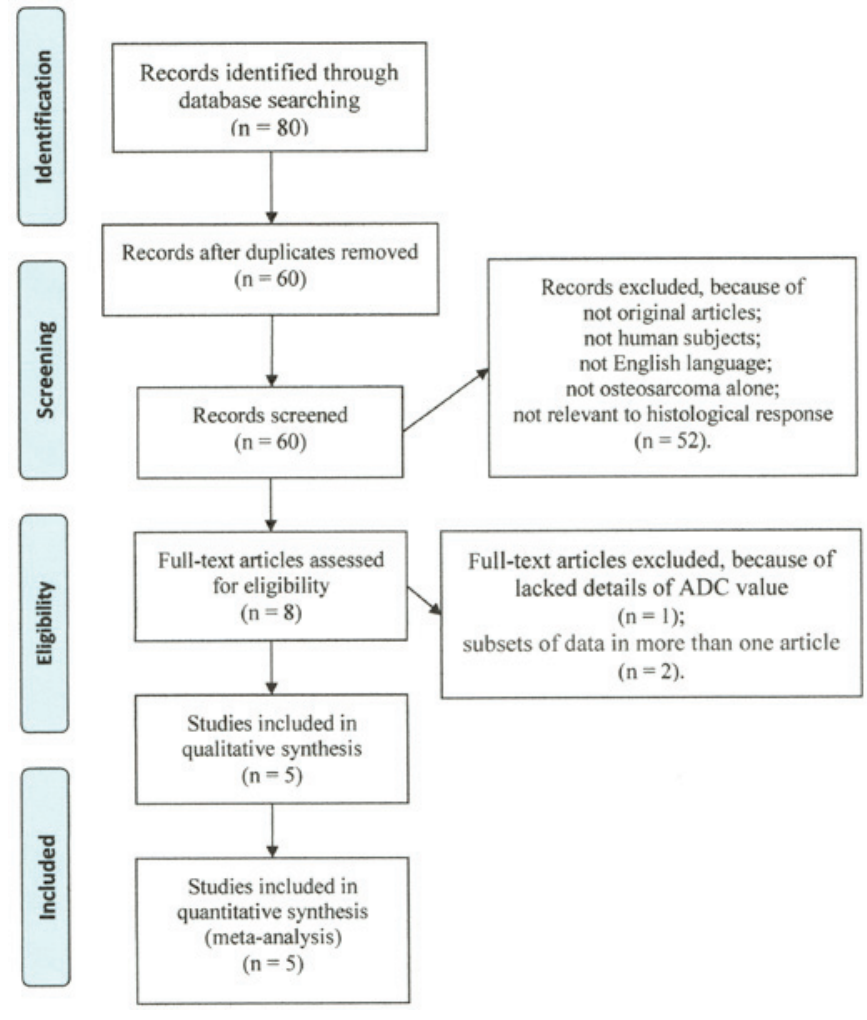

Figure 1. A flowchart of the study-selection procedure. ADC, apparent diffusion coefficient.

the combination of chemotherapy and surgery; I, preoperative DWI assessment for chemotherapy response; C, histological assessment for chemotherapy response; $\mathrm{O}$, mean difference of $\mathrm{ADC}$ and $\mathrm{ADC}$ ratio; $\mathrm{S}$, retrospective and prospective cohort studies. Using the predefined electric literature databases, we identified 80 potentially eligible articles, of which 72 were excluded due to duplication or after reviewing the article title and abstract. Subsequently, 3 articles were excluded after reviewing the whole article (23-25). Five articles with 106 patients who fulfilled all of the inclusion criteria were selected for the meta-analysis (Table I) (26-30). The detailed procedure of study selection and its exclusion reasons in the meta-analysis is presented in Fig. 1.

Study description and quality evaluation. Table II presents the clinical characteristics of the 5 studies included in the meta-analysis. All included studies fulfilled $\geq 5$ 'low' answers in the 7 domains of the QUADAS-2 tool for methodological quality assessment. Common weaknesses concentrated on the domain of patient selection (Table III).

Meta-analysis. There was significant heterogeneity among the 5 studies in terms of the mean difference in ADC between good and poor responders $\left(\mathrm{P}=0.0004\right.$ and $\left.\mathrm{I}^{2}=80 \%\right)$. Therefore, the random effect model was used. Significant mean differences were identified between good and poor responders in the ADC value (mean difference, 0.33; 95\% CI, 0.04-0.63; $\mathrm{P}=0.03$ ). The good responders had a higher ADC value than the poor responders (Fig. 2A).

The mean difference in ADC ratio between good and poor responders was calculated with the fixed effects model, as 
Table I. Summary of the studies included in the meta-analyses.

\begin{tabular}{|c|c|c|c|c|c|c|c|}
\hline $\begin{array}{l}\text { First } \\
\text { author }\end{array}$ & Year & Journal & Country & $\mathrm{N}$ & Study design & Enrolment & (Refs.) \\
\hline Baunin & 2012 & Skeletal radiol & France & 14 & Prospective & N/D & (26) \\
\hline Byun & 2013 & J. Nucl med & Korea & 27 & Prospective & Consecutive & (27) \\
\hline Oka & 2010 & Skeletal radiol & Japan & 22 & Retrospective & N/A & (28) \\
\hline Uhl & 2006 & Pediatr radiol & Germany & 8 & Prospective & N/D & (29) \\
\hline Wang & 2013 & PLoS One & China & 35 & Prospective & N/D & (30) \\
\hline
\end{tabular}

N/D, not documented; N/A, not available; N, number of patients.

Table II. Clinical characteristics of the patients included in the meta-analysis.

\begin{tabular}{lccccccr}
\hline $\begin{array}{l}\text { First } \\
\text { author }\end{array}$ & Year & M/F & $\begin{array}{c}\text { Age, years } \\
\text { (mean/range) }\end{array}$ & $\begin{array}{c}\text { Field, } \\
\text { strength tesla }\end{array}$ & $\begin{array}{c}\text { b-values, } \\
\text { s/mm }\end{array}$ & Assessors & Blindness \\
Baunin & 2012 & N/D & N/D & N/D & 0,900 & 2 & N/D \\
Byun & 2013 & $15 / 12$ & $20.6 / 14-23$ & 3.0 & 0,800 & 2 & N/D \\
Oka & 2010 & $8 / 14$ & $15.3 / 8-29$ & 1.5 & 0,1000 & 4 & Blind \\
Uhl & 2006 & N/D & $14.7 / 11-19$ & 1.5 & 0,700 & 2 & Blind \\
Wang & 2013 & $18 / 17$ & $26.8 / 7-65$ & 1.5 & 0,700 & 2 & N/D \\
\hline
\end{tabular}

N/D, not documented; M/F, male/female.

Table III. Quality assessment of diagnostic accuracy studies-2.

\begin{tabular}{|c|c|c|c|c|c|c|c|c|}
\hline \multirow[b]{2}{*}{$\begin{array}{l}\text { First } \\
\text { author }\end{array}$} & \multicolumn{4}{|c|}{ Risk of bias } & \multicolumn{3}{|c|}{ Applicability concerns } & \multirow[b]{2}{*}{ (Refs.) } \\
\hline & $\begin{array}{l}\text { Patient } \\
\text { selection }\end{array}$ & $\begin{array}{l}\text { Index } \\
\text { test }\end{array}$ & $\begin{array}{c}\text { Reference } \\
\text { standard }\end{array}$ & $\begin{array}{l}\text { Flow and } \\
\text { timing }\end{array}$ & $\begin{array}{l}\text { Patient } \\
\text { selection }\end{array}$ & $\begin{array}{l}\text { Index } \\
\text { test }\end{array}$ & $\begin{array}{c}\text { Reference } \\
\text { standard }\end{array}$ & \\
\hline Baunin & Unclear risk & Low risk & Low risk & Low risk & Unclear risk & Low risk & Low risk & (26) \\
\hline Byun & Low risk & Low risk & Low risk & Low risk & Low risk & Low risk & Low risk & $(27)$ \\
\hline Oka & High risk & Low risk & Low risk & Low risk & High risk & Low risk & Low risk & (28) \\
\hline Uhl & Unclear risk & Low risk & Low risk & Low risk & Unclear risk & Low risk & Low risk & $(29)$ \\
\hline Wang & Unclear risk & Low risk & Low risk & Low risk & Unclear risk & Low risk & Low risk & $(30)$ \\
\hline
\end{tabular}

there was no heterogeneity among the studies $\left(\mathrm{P}=0.88 ; \mathrm{I}^{2}=0 \%\right)$. There was a significant mean difference between the good and poor responders in the ADC ratio of the 3 studies (mean difference, 21.3; 95\% CI, 12.2-30.5; $\mathrm{P}<0.00001$ ) (Fig. 2B).

Publication bias was assessed using funnel plot asymmetry tests (Fig. 3). The plot of ADC meta-analysis among the 5 studies was asymmetric, indicating that there was some possible publication bias. However, the plots of ADC ratio meta-analysis was symmetric, suggesting a low risk of publication bias.

\section{Discussion}

Certain studies revealed that an increased ADC following neoadjuvant chemotherapy is associated with good histological response $(24,25,27,29,30)$. However, there have been conflicting results. Other studies have not identified a significant association between ADC value and tumor necrosis $(23,26,28)$. Therefore, the predictive value of ADC remained undetermined. This meta-analysis focused on not only the ADC but also the ADC ratio for evaluating the chemotherapy response, which to the best of our knowledge had not previously been studied. The present study found that the good responders demonstrated a higher ADC and a higher ADC ratio than the poor responders.

The current study has several limitations. Firstly, only 3 articles with 44 patients were selected for the ADC ratio study. Further studies based on these promising results are warranted. Secondly, it was not possible to completely exclude potential bias, despite the following efforts. To minimize bias in the study selection and the data extraction, this study was 


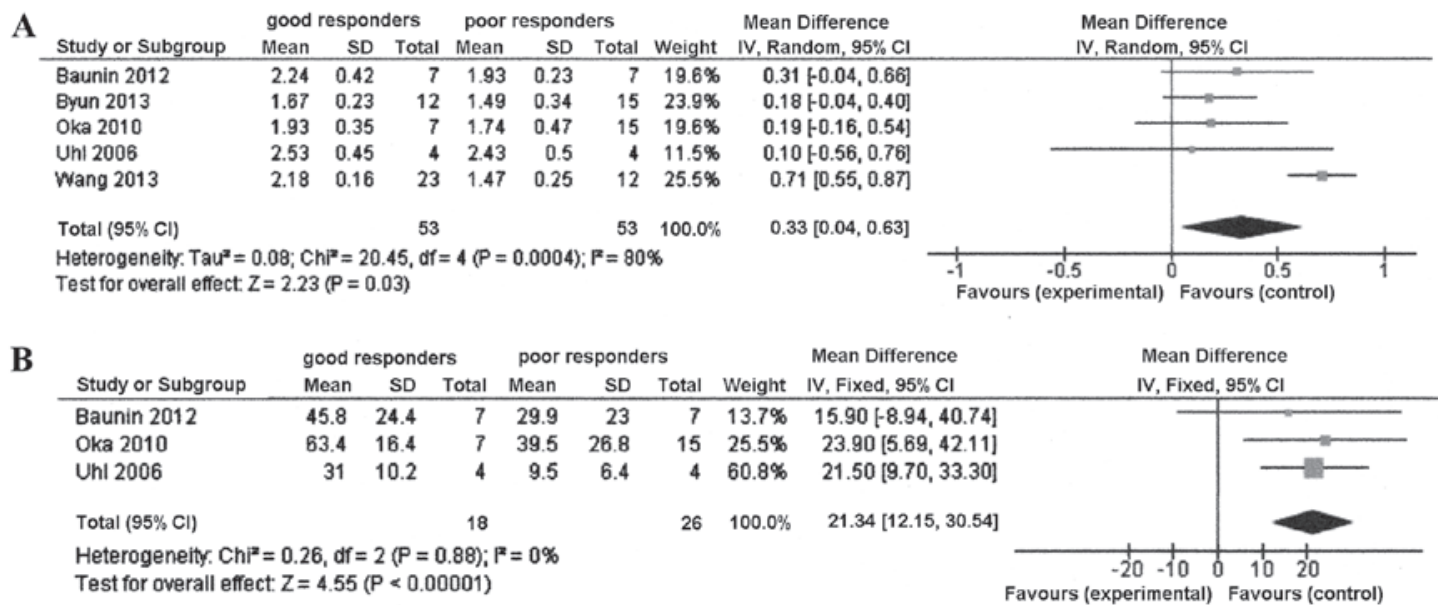

Figure 2. Forest plot showing outcomes of (A) the mean difference of ADC between good and poor responders in all 5 studies and (B) ADC ratio between good and poor responders in the 3 studies. Heterogeneity among studies was also evaluated using the $\chi^{2}$ statistical test and the inconsistency index $\mathrm{I}^{2}$. ADC, apparent diffusion coefficient; SD, standard deviation; $\mathrm{CI}$, confidence interval.
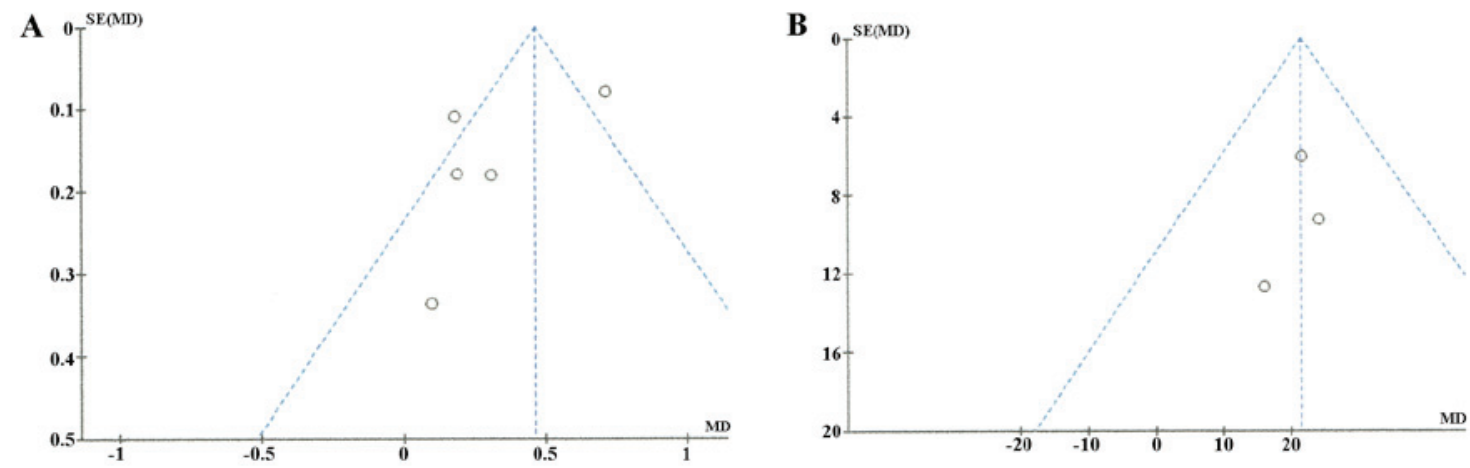

Figure 3. Funnel plot of the diagnostic odds ratios of (A) all 5 ADC articles and (B) the 3 ADC ratio articles. ADC, apparent diffusion coefficient.

performed blindly and independently. To be sure that all the selected articles were high-quality, only articles with a 'low' answer for the 7 domains in the QUADAS-2 quality assessment tool of $\geq 5$ were selected. Publication bias was also assessed using a funnel plot, and there was some possible publication bias in the ADC meta-analysis among the 5 studies. Further prospective assessment of DWI to evaluate the chemotherapeutic response of osteosarcoma is required in order to exclude potential bias completely. Thirdly, significantly high heterogeneity of the diagnostic performance of DWI was found in the ADC meta-analysis among the 5 studies. This heterogeneity might be attributable to the methodological differences between the articles, such as the DWI acquisition technique, interpretation scheme or reference standard. For example, there was variation in the combinations of b-value and in the choice of region of interest among the 5 studies. Additional investigations with larger cohorts and the methodology of MR techniques that are both standardized and optimized are necessary to better characterize the benefit of this new technology for patients with osteosarcoma.

Recently, PET or PET/CT has become one of the most intensively-investigated imaging modalities for the monitoring of preoperative chemotherapy effects (12). Byun et al (27) reported the equivalent potential of PET/CT and DWI to predict the histologic response to neoadjuvant chemotherapy in 28 patients with extremity osteosarcoma, using sequential imaging capture. The combination of PET/CT and DWI may yield varied biological information, such as changes in glucose metabolism and cellularity, which means that it has the potential to overcome the functional limitations of individual PET/CT and DWI. Several studies have suggested $40-60 \%$ of the standardized uptake value (SUV) ratio as the PET/CT cutoff point for a good response to neoadjuvant chemotherapy of osteosarcoma $(12,27,31)$, but the optimal ADC cutoff point for good responders to neoadjuvant chemotherapy of osteosarcoma has yet to be reported. Furthermore, there is no standard means to measure ADC values. Further studies are required in order to obtain the standardized and optimized ADC values of DWI.

In conclusion, the present meta-analysis has demonstrated that the ADC and ADC ratio are useful for predicting the histologic response of patients to preoperative chemotherapy in osteosarcoma. This method may have promising potential as a preoperative non-invasive modality. For poor responders to preoperative chemotherapy, a more radical tumor resection should be performed and the postoperative chemotherapeutic regimen should be altered. For good responders, a minimally invasive surgical procedure can be selected with a low risk of local recurrence. These results have the potential to change the present therapeutic strategy for osteosarcoma based on the role of DWI prior to and following adjuvant chemotherapy. 


\section{Acknowledgements}

The present study is supported by the Practical Research for Innovative Cancer Control from Japan Agency For Medical Research and development (AMED) and Japan Society for the Promotion of Science (JSPS) KAKENHI (grant no. 26462267).

\section{References}

1. Bacci G, Briccoli A, Ferrari S, Longhi A, Mercuri M, Capanna R, Donati D, Lari S, Forni C and DePaolis M: Neoadjuvant chemotherapy for osteosarcoma of the extremity: Long-term results of the Rizzoli's 4th protocol. Eur J Cancer 37: 2030-2039, 2001.

2. Bielack SS, Kempf-Bielack B, Delling G, Exner GU, Flege S, Helmke K, Kotz R, Salzer-Kuntschik M, Werner M, Winkelmann W, et al: Prognostic factors in high-grade osteosarcoma of the extremities or trunk: An analysis of 1,702 patients treated on neoadjuvant cooperative osteosarcoma study group protocols. J Clin Oncol 20: 776-790, 2002.

3. Lewis IJ, Nooij MA, Whelan J, Sydes MR, Grimer R, Hogendoorn PC, Memon MA, Weeden S, Uscinska BM, van Glabbeke M, et al: Improvement in histologic response but not survival in osteosarcoma patients treated with intensified chemotherapy: A randomized phase III trial of the European Osteosarcoma Intergroup. J Natl Cancer Inst 99: 112-128, 2007.

4. Eselgrim M, Grunert H, Kühne T, Zoubek A, Kevric M, Bürger H, Jürgens H, Mayer-Steinacker R, Gosheger G and Bielack SS: Dose intensity of chemotherapy for osteosarcoma and outcome in the Cooperative Osteosarcoma Study Group (COSS) trials. Pediatr Blood Cancer 47: 42-50, 2006.

5. Meyers PA, Gorlick R, Heller G, Casper E, Lane J, Huvos AG and Healey JH: Intensification of preoperative chemotherapy for osteogenic sarcoma: Results of the Memorial Sloan-Kettering (T12) protocol. J Clin Oncol 16: 2452-2458, 1998.

6. Huvos AG, Rosen G and Marcove RC: Primary osteogenic sarcoma: Pathologic aspects in 20 patients after treatment with chemotherapy en bloc resection, and prosthetic bone replacement. Arch Pathol Lab Med 101: 14-18, 1977.

7. Picci P, Bacci G, Campanacci M, Gasparini M, Pilotti S, Cerasoli S, Bertoni F, Guerra A, Capanna R, Albisinni U, et al: Histologic evaluation of necrosis in osteosarcoma induced by chemotherapy. Regional mapping of viable and nonviable tumor Cancer 56: 1515-1521, 1985.

8. Guo J, Reddick WE, Glass JO, Ji Q, Billups CA, Wu J, Hoffer FA, Kaste SC, Jenkins JJ, Ortega Flores XC, et al: Dynamic contrast-enhanced magnetic resonance imaging as a prognostic factor in predicting event-free and overall survival in pediatric patients with osteosarcoma. Cancer 118: 3776-3785, 2012.

9. Kubo T, Furuta T, Johan MP, Adachi N and Ochi M: Percent slope analysis of dynamic magnetic resonance imaging for assessment of chemotherapy response of osteosarcoma or Ewing sarcoma: Systematic review and meta-analysis. Skeletal Radiol 45: 1235-1242, 2016.

10. Inaki A, Taki J, Wakabayashi H, Sumiya H, Zen Y, Tsuchiya H and Kinuya S: Thallium-201 scintigraphy for the assessment of long-term prognosis in patients with osteosarcoma. Ann Nucl Med 26: 545-550, 2012.

11. Kubo T, Shimose S, Fujimori J,Furuta T and Ochi M: Quantitative (201)thallium scintigraphy for prediction of histological response to neoadjuvant chemotherapy in osteosarcoma; systematic review and meta-analysis. Surg Oncol 24: 194-199, 2015.

12. Hongtao L, Hui Z, Bingshun W, Xiaojin W, Zhiyu W, Shuier Z, Aina H, Yuanjue S, Daliu M, Zan S and Yang Y: 18 F-FDG positron emission tomography for the assessment of histological response to neoadjuvant chemotherapy in osteosarcomas: A meta-analysis. Surg Oncol 21: e165-e170, 2012.

13. van Rijswijk CS, Kunz P, Hogendoorn PC, Taminiau AH, Doornbos J and Bloem JL: Diffusion-weighted MRI in the characterization of soft-tissue tumors. J Magn Reson Imaging 15: 302-307, 2002.

14. Baur A, Huber A, Arbogast S, Dürr HR, Zysk S, Wendtner C, Deimling $M$ and Reiser M: Diffusion-weighted imaging of tumor recurrencies and posttherapeutical soft-tissue changes in humans. Eur Radiol 11: 828-833, 2001.

15. Herneth AM, Friedrich K, Weidekamm C, Schibany N, Krestan C, Czerny $\mathrm{C}$ and Kainberger F: Diffusion weighted imaging of bone marrow pathologies. Eur J Radiol 55: 74-83, 2005.
16. MacKenzie JD, Gonzalez L, Hernandez A, Ruppert K and Jaramillo D: Diffusion-weighted and diffusion tensor imaging for pediatric musculoskeletal disorders. Pediatr Radiol 37: 781-788, 2007.

17. Nonomura Y, Yasumoto M, Yoshimura R, Haraguchi K, Ito S, Akashi $\mathrm{T}$ and Ohashi I: Relationship between bone marrow cellularity and apparent diffusion coefficient. J Magn Reson Imaging 13: 757-760, 2001.

18. Humphries PD, Sebire NJ, Siegel MJ and Olsen ØE: Tumors in pediatric patients at diffusion-weighted MR imaging: Apparent diffusion coefficient and tumor cellularity. Radiology 245: 848-854, 2007

19. Lang P, Wendland MF, Saeed M, Gindele A, Rosenau W, Mathur A, Gooding CA and Genant HK: Osteogenic osteosarcoma: Noninvasive in vivo assessment of tumor necrosis with diffusion-weighted MR imaging. Radiology 206: 227-235, 1998.

20. Thoeny HC, De Keyzer F, Chen F, Ni Y, Landuyt W, Verbeken EK, Bosmans H, Marchal G and Hermans R: Diffusion-weighted MR imaging in monitoring the effect of a vascular targeting agent on rhabdomyosarcoma in rats. Radiology 234: 756-764, 2005.

21. Liberati A, Altman DG, Tetzlaff J, Mulrow C, Gøtzsche PC, Ioannidis JP, Clarke M, Devereaux PJ, Kleijnen J and Moher D: The PRISMA statement for reporting systematic reviews and meta-analyses of studies that evaluate health care interventions: Explanation and elaboration. PLoS Med 6: e1000100, 2009.

22. Whiting PF, Rutjes AW, Westwood ME, Mallett S, Deeks JJ, Reitsma JB, Leeflang MM, Sterne JA and Bossuyt PM: QUADAS-2: A revised tool for the quality assessment of diagnostic accuracy studies. Ann Intern Med 155: 529-536, 2011.

23. Bajpai J, Gamnagatti S, Kumar R, Sreenivas V, Sharma MC, Khan SA, Rastogi S, Malhotra A, Safaya R and Bakhshi S: Role of MRI in osteosarcoma for evaluation and prediction of chemotherapy response: Correlation with histological necrosis. Pediatr Radiol 41: 441-450, 2011.

24. Hayashida Y, Yakushiji T, Awai K, Katahira K, Nakayama Y, Shimomura O, Kitajima M, Hirai T, Yamashita Y and Mizuta H: Monitoring therapeutic responses of primary bone tumors by diffusion-weighted image: Initial results. Eur Radiol 16: 2637-2643, 2006

25. Uhl M, Saueressig U, van Buiren M, Kontny U, Niemeyer C, Köhler G, Ilyasov K and Langer M: Osteosarcoma: Preliminary results of in vivo assessment of tumor necrosis after chemotherapy with diffusion- and perfusion-weighted magnetic resonance imaging. Invest Radiol 41: 618-623, 2006.

26. Baunin C, Schmidt G, Baumstarck K, Bouvier C, Gentet JC, Aschero A, Ruocco A, Bourlière B, Gorincour G, Desvignes C, et al: Value of diffusion-weighted images in differentiating mid-course responders to chemotherapy for osteosarcoma compared to the histological response: Preliminary results. Skeletal Radiol 41: 1141-1149, 2012.

27. Byun BH, Kong CB, Lim I, Choi CW, Song WS, Cho WH, Jeon DG, Koh JS, Lee SY and Lim SM: Combination of 18F-FDG PET/CT and diffusion-weighted MR imaging as a predictor of histologic response to neoadjuvant chemotherapy: Preliminary results in osteosarcoma. J Nucl Med 54: 1053-1059, 2013.

28. Oka K, Yakushiji T, Sato H, Hirai T, Yamashita Y and Mizuta H: The value of diffusion-weighted imaging for monitoring the chemotherapeutic response of osteosarcoma: A comparison between average apparent diffusion coefficient and minimum apparent diffusion coefficient. Skeletal Radiol 39: 141-146, 2010.

29. Uhl M, Saueressig U, Koehler G, Kontny U, Niemeyer C, Reichardt W, Ilyasof K, Bley T and Langer M: Evaluation of tumour necrosis during chemotherapy with diffusion-weighted MR imaging: Preliminary results in osteosarcomas. Pediatr Radiol 36: 1306-1311, 2006.

30. Wang CS, Du LJ, Si MJ, Yin QH, Chen L, Shu M, Yuan F, Fei XC and Ding XY: Noninvasive assessment of response to neoadjuvant chemotherapy in osteosarcoma of long bones with diffusion-weighted imaging: An initial in vivo study. PLoS One 8: e72679, 2013

31. Im HJ, Kim TS, Park SY, Min HS, Kim JH, Kang HG, Park SE Kwon MM, Yoon JH, Park HJ, et al: Prediction of tumour necrosis fractions using metabolic and volumetric 18F-FDG PET/CT indices, after one course and at the completion of neoadjuvant chemotherapy, in children and young adults with osteosarcoma. Eur J Nucl Med Mol Imaging 39: 39-49, 2012. 